\title{
The Relationship between Focal Surfaces and Surfaces at a Constant Distance from the Edge of Regression on a Surface
}

\author{
Semra Yurttancikmaz and Omer Tarakci \\ Department of Mathematics, Faculty of Science, Ataturk University, 25240 Erzurum, Turkey \\ Correspondence should be addressed to Semra Yurttancikmaz; semrakaya@atauni.edu.tr
}

Received 7 July 2014; Accepted 8 September 2014

Academic Editor: John D. Clayton

Copyright ( $) 2015$ S. Yurttancikmaz and O. Tarakci. This is an open access article distributed under the Creative Commons Attribution License, which permits unrestricted use, distribution, and reproduction in any medium, provided the original work is properly cited.

\begin{abstract}
We investigate the relationship between focal surfaces and surfaces at a constant distance from the edge of regression on a surface. We show that focal surfaces $F_{1}$ and $F_{2}$ of the surface $M$ can be obtained by means of some special surfaces at a constant distance from the edge of regression on the surface $M$.
\end{abstract}

\section{Introduction}

Surfaces at a constant distance from the edge of regression on a surface were firstly defined by Tarakci in 2002 [1]. These surfaces were obtained by taking a surface instead of a curve in the study suggested by Hans Vogler in 1963. In the mentioned study, Hans Vogler asserted notion of curve at a constant distance from the edge of regression on a curve. Also, Tarakci and Hacisalihoglu calculated some properties and theorems which known for parallel surfaces for surfaces at a constant distance from the edge of regression on a surface [2]. Later, various authors became interested in surfaces at a constant distance from the edge of regression on a surface and investigated Euler theorem and Dupin indicatrix, conjugate tangent vectors, and asymptotic directions for this surface [3] and examined surfaces at a constant distance from the edge of regression on a surface in $E_{1}^{3}$ Minkowski space [4].

Another issue that we will use in this paper is the focal surface. Focal surfaces are known in the field of line congruence. Line congruence has been introduced in the field of visualization by Hagen et al. in 1991 [5]. They can be used to visualize the pressure and heat distribution on an airplane, temperature, rainfall, ozone over the earth's surface, and so forth. Focal surfaces are also used as a surface interrogation tool to analyse the "quality" of the surface before further processing of the surface, for example, in a NC-milling operation [6]. Generalized focal surfaces are related to hedgehog diagrams. Instead of drawing surface normals proportional to a surface value, only the point on the surface normal proportional to the function is drawing. The loci of all these points are the generalized focal surface. This method was introduced by Hagen and Hahmann $[6,7]$ and is based on the concept of focal surface which is known from line geometry. The focal surfaces are the loci of all focal points of special congruence, the normal congruence. In later years, focal surfaces have been studied by various authors in different fields.

In this paper, we have discovered a new method to constitute focal surfaces by means of surfaces at a constant distance from the edge of regression on a surface. Focal surfaces $F_{1}$ and $F_{2}$ of the surface $M$ in $E^{3}$ are associated with surfaces at a constant distance from the edge of regression on $M$ that formed along directions of $Z_{P}$ lying in planes $S p\left\{\phi_{u}, N\right\}$ and $S p\left\{\phi_{v}, N\right\}$, respectively.

\section{Surfaces at a Constant Distance from the Edge of Regression on a Surface}

Definition 1. Let $M$ and $M^{f}$ be two surfaces in $E^{3}$ Euclidean space and let $N_{P}$ be a unit normal vector and let $T_{P} M$ be tangent space at point $P$ of surface $M$ and let $\left\{X_{P}, Y_{P}\right\}$ be orthonormal bases of $T_{P} M$. Take a unit vector $Z_{P}=d_{1} X_{P}+$ 
$d_{2} Y_{P}+d_{3} N_{P}$, where $d_{1}, d_{2}, d_{3} \in \mathbb{R}$ are constant and $d_{1}^{2}+d_{2}^{2}+$ $d_{3}^{2}=1$. If there is a function $f$ defined by

$$
f: M \longrightarrow M^{f}, \quad f(P)=P+r Z_{P},
$$

where $r \in \mathbb{R}$, then the surface $M^{f}$ is called the surface at a constant distance from the edge of regression on the surface M.

Here, if $d_{1}=d_{2}=0$, then $Z_{P}=N_{P}$ and so $M$ and $M^{f}$ are parallel surfaces. Now, we represent parametrization of surfaces at a constant distance from the edge of regression on $M$. Let $(\phi, U)$ be a parametrization of $M$, so we can write that

$$
\begin{gathered}
\phi: U \subset E^{2} \longrightarrow M \\
(u, v) \quad \phi(u, v) .
\end{gathered}
$$

In case $\left\{\phi_{u}, \phi_{v}\right\}$ is a basis of $T_{P} M$, then we can write that $Z_{P}=d_{1} \phi_{u}+d_{2} \phi_{v}+d_{3} N_{P}$, where $\phi_{u}, \phi_{v}$ are, respectively, partial derivatives of $\phi$ according to $u$ and $v$. Since $M^{f}=\{f(P)$ : $\left.f(P)=P+r Z_{P}\right\}$, a parametric representation of $M^{f}$ is

$$
\psi(u, v)=\phi(u, v)+r Z(u, v) .
$$

Thus, it is obtained that

$$
\begin{aligned}
M^{f}=\{\psi(u, v): & \psi(u, v) \\
= & \phi(u, v) \\
& +r\left(d_{1} \phi_{u}(u, v)\right. \\
& \quad+d_{2} \phi_{v}(u, v) \\
& \left.\left.\quad+d_{3} N(u, v)\right)\right\}
\end{aligned}
$$

and if we get $r d_{1}=\lambda_{1}, r d_{2}=\lambda_{2}, r d_{3}=\lambda_{3}$, then we have

$$
\begin{aligned}
M^{f}=\{\psi(u, v): & \psi(u, v) \\
= & \phi(u, v)+\lambda_{1} \phi_{u}(u, v) \\
& +\lambda_{2} \phi_{v}(u, v)+\lambda_{3} N(u, v), \\
& \left.\lambda_{1}^{2}+\lambda_{2}^{2}+\lambda_{3}^{2}=r^{2}\right\} .
\end{aligned}
$$

Calculation of $\psi_{u}$ and $\psi_{v}$ gives us that

$$
\begin{aligned}
& \psi_{u}=\phi_{u}+\lambda_{1} \phi_{u u}+\lambda_{2} \phi_{v u}+\lambda_{3} N_{u}, \\
& \psi_{v}=\phi_{v}+\lambda_{1} \phi_{u v}+\lambda_{2} \phi_{v v}+\lambda_{3} N_{v} .
\end{aligned}
$$

Here, $\phi_{u u}, \phi_{v u}, \phi_{u v}, \phi_{v v}, N_{u}, N_{v}$ are calculated as in [1]. We choose curvature lines instead of parameter curves of $M$ and let $u$ and $v$ be arc length of these curvature lines. Thus, the following equations are obtained:

$$
\begin{aligned}
\phi_{u u} & =-\kappa_{1} N, \\
\phi_{v v} & =-\kappa_{2} N, \\
\phi_{u v} & =\phi_{v u}=0, \\
N_{u} & =\kappa_{1} \phi_{u}, \\
N_{v} & =\kappa_{2} \phi_{v} .
\end{aligned}
$$

From (6) and (7), we find

$$
\begin{aligned}
& \psi_{u}=\left(1+\lambda_{3} \kappa_{1}\right) \phi_{u}-\lambda_{1} \kappa_{1} N, \\
& \psi_{v}=\left(1+\lambda_{3} \kappa_{2}\right) \phi_{v}-\lambda_{2} \kappa_{2} N
\end{aligned}
$$

and $\left\{\psi_{u}, \psi_{v}\right\}$ is a basis of $\chi\left(M^{f}\right)$. If we denote by $N^{f}$ unit normal vector of $M^{f}$, then $N^{f}$ is

$$
\begin{aligned}
N^{f}= & \frac{\left[\psi_{u}, \psi_{v}\right]}{\left\|\left[\psi_{u}, \psi_{v}\right]\right\|} \\
= & \left(\lambda_{1} \kappa_{1}\left(1+\lambda_{3} \kappa_{2}\right) \phi_{u}+\lambda_{2} \kappa_{2}\left(1+\lambda_{3} \kappa_{1}\right) \phi_{v}\right. \\
& \left.+\left(1+\lambda_{3} \kappa_{1}\right)\left(1+\lambda_{3} \kappa_{2}\right) N\right) \\
& \times\left(\lambda_{1}^{2} \kappa_{1}^{2}\left(1+\lambda_{3} \kappa_{2}\right)^{2}+\lambda_{2}^{2} \kappa_{2}^{2}\left(1+\lambda_{3} \kappa_{1}\right)^{2}\right. \\
& \left.+\left(1+\lambda_{3} \kappa_{1}\right)^{2}\left(1+\lambda_{3} \kappa_{2}\right)^{2}\right)^{-1 / 2},
\end{aligned}
$$

where $\kappa_{1}, \kappa_{2}$ are principal curvatures of the surface $M$. If

$$
\begin{gathered}
A=\left(\lambda_{1}^{2} \kappa_{1}^{2}\left(1+\lambda_{3} \kappa_{2}\right)^{2}+\lambda_{2}^{2} \kappa_{2}^{2}\left(1+\lambda_{3} \kappa_{1}\right)^{2}\right. \\
\left.+\left(1+\lambda_{3} \kappa_{1}\right)^{2}\left(1+\lambda_{3} \kappa_{2}\right)^{2}\right)^{1 / 2}
\end{gathered}
$$

we can write

$$
\begin{aligned}
N^{f}= & \frac{\lambda_{1} \kappa_{1}\left(1+\lambda_{3} \kappa_{2}\right)}{A} \phi_{u}+\frac{\lambda_{2} \kappa_{2}\left(1+\lambda_{3} \kappa_{1}\right)}{A} \phi_{v} \\
& +\frac{\left(1+\lambda_{3} \kappa_{1}\right)\left(1+\lambda_{3} \kappa_{2}\right)}{A} N .
\end{aligned}
$$

Here, in case of $\kappa_{1}=\kappa_{2}$ and $\lambda_{3}=-1 / \kappa_{1}=-1 / \kappa_{2}$ since $\psi_{u}$ and $\psi_{v}$ are not linearly independent, $M^{f}$ is not a regular surface. We will not consider this case [1].

\section{Focal Surfaces}

The differential geometry of smooth three-dimensional surfaces can be interpreted from one of two perspectives: in terms of oriented frames located on the surface or in terms of a pair of associated focal surfaces. These focal surfaces are swept by the loci of the principal curvatures radii. Considering fundamental facts from differential geometry, it is obvious that the centers of curvature of the normal section curves at a particular point on the surface fill out a certain segment of the normal vector at this point. The extremities of these segments are the centers of curvature of two principal directions. These two points are called the focal points of this particular normal [8]. This terminology is justified by the fact that a line congruence can be considered as the set of lines touching two surfaces, the focal surfaces of the line congruence. The points of contact between a line of the congruence and the two focal surfaces are the focal points of this line. It turns out that the focal points of a normal congruence are the centers of curvature of the two principal directions $[9,10]$. 
We represent surfaces parametrically as vector-valued functions $\phi(u, v)$. Given a set of unit vectors $Z(u, v)$, a line congruence is defined:

$$
C(u, v)=\phi(u, v)+D(u, v) Z(u, v)
$$

where $D(u, v)$ is called the signed distance between $\phi(u, v)$ and $Z(u, v)$ [8]. Let $N(u, v)$ be unit normal vector of the surface. If $Z(u, v)=N(u, v)$, then $C=C_{N}$ is a normal congruence. A focal surface is a special normal congruence. The parametric representation of the focal surfaces of $C_{N}$ is given by

$$
F_{i}(u, v)=\phi(u, v)-\frac{1}{\kappa_{i}(u, v)} N(u, v) ; \quad i=1,2,
$$

where $\kappa_{1}, \kappa_{2}$ are the principal curvatures. Except for parabolic points and planar points where one or both principal curvatures are zero, each point on the base surface is associated with two focal points. Thus, generally, a smooth base surface has two focal surface sheets, $F_{1}(u, v)$ and $F_{2}(u, v)$ [11].

The generalization of this classical concept leads to the generalized focal surfaces:

$$
F(u, v)=\phi(u, v)+a f\left(\kappa_{1}, \kappa_{2}\right) N(u, v) \quad \text { with } a \in \mathbb{R}
$$

where the scalar function $f$ depends on the principal curvatures $\kappa_{1}=\kappa_{1}(u, v)$ and $\kappa_{2}=\kappa_{2}(u, v)$ of the surface $M$. The real number $a$ is used as a scale factor. If the curvatures are very small you need a very large number $a$ to distinguish the two surfaces $\phi(u, v)$ and $F(u, v)$ on the screen. Variation of this factor can also improve the visibility of several properties of the focal surface; for example, one can get intersections clearer [6].

\section{The Relationship between Focal Surfaces and Surfaces at a Constant Distance from the Edge of Regression on a Surface}

Theorem 2. Let surface $M$ be given by parametrical $\phi(u, v)$. One considers all surfaces at a constant distance from the edge of regression on $M$ that formed along directions of $Z_{P}$ lying in plane $S p\left\{\phi_{u}, N\right\}$. Normals of these surfaces at points $f(P)$ corresponding to point $P \in M$ generate a spatial family of line of which top is center of first principal curvature $C_{1}=P-$ $\left(1 / \kappa_{1}(P)\right) N_{P}$ at $P$.

Proof. Surfaces at a constant distance from the edge of regression on $M$ that formed along directions of $Z_{P}$ lying in plane $S p\left\{\phi_{u}, N\right\}$ are defined by

$$
\begin{aligned}
& f_{i}: M \longrightarrow M^{f_{i}}, \quad i=1,2, \ldots, \\
& f_{i}(P)=P+\lambda_{1_{i}} \phi_{u}(P)+\lambda_{3_{i}} N_{P} .
\end{aligned}
$$

These surfaces and their unit normal vectors are, respectively, denoted by $M^{f_{i}}$ and $N^{f_{i}}$. We will demonstrate that intersection point of lines which pass from the point $f_{i}(P)$ and are in direction $N_{f_{i}(P)}^{f_{i}}$ is $C_{1}=P-\left(1 / \kappa_{1}(P)\right) N_{P}$.
The normal vector of the surface $M^{f_{i}}$ at the point $f_{i}(P)$ is

$$
N^{f_{i}}=\lambda_{1_{i}} \kappa_{1}(P) \phi_{u}(P)+\left(1+\lambda_{3_{i}} \kappa_{1}(P)\right) N_{P} .
$$

Here, it is clear that $N^{f_{i}}$ is in plane $S p\left\{\phi_{u}, N\right\}$. Suppose that line passing from the point $f_{i}(P)$ and being in direction $N_{f_{i}(P)}^{f_{i}}$ is $d_{i}$ and a representative point of $d_{i}$ is $Q=(x, y)=x \phi_{u}(P)+$ $y N_{P}$; then, the equation of $d_{i}$ is

$$
d_{i} \cdots \overrightarrow{P Q}=\overrightarrow{P f_{i}(P)}+\mu_{1} N_{f_{i}(P)}^{f_{i}}
$$

Besides, suppose that line passing from the point $f_{j}(P)$ and being in direction $N_{f_{j}(P)}^{f_{j}}$ is $d_{j}$ and a representative point of $d_{j}$ is $R=(x, y)$; then, equation of $d_{j}$ is

$$
d_{j} \cdots \overrightarrow{P R}=\overrightarrow{P f_{j}(P)}+\mu_{2} N_{f_{j}(P)}^{f_{j}}, \quad j=1,2, \ldots
$$

We find intersection point of these lines. Since it is studied in plane of vectors $\left\{\phi_{u}(P), N_{P}\right\}$, the point $P$ can be taken as beginning point. If we arrange the lines $d_{i}$ and $d_{j}$, then we find

$$
\begin{aligned}
d_{i} \cdots(x, y) & =\left(\lambda_{1_{i}}, \lambda_{3_{i}}\right)+\mu_{1}\left(\lambda_{1_{i}} \kappa_{1}, 1+\lambda_{3_{i}} \kappa_{1}\right), \\
d_{i} \cdots y & =\frac{1+\lambda_{3_{i}} \kappa_{1}}{\lambda_{1_{i}} \kappa_{1}} x-\frac{1}{\kappa_{1}}, \\
d_{j} \cdots(x, y) & =\left(\lambda_{1_{j}}, \lambda_{3_{j}}\right)+\mu_{2}\left(\lambda_{1_{j}} \kappa_{1}, 1+\lambda_{3_{j}} \kappa_{1}\right), \\
d_{j} \cdots y & =\frac{1+\lambda_{3_{j}} \kappa_{1}}{\lambda_{1 j} \kappa_{1}} x-\frac{1}{\kappa_{1}} .
\end{aligned}
$$

From here, it is clear that intersection point of $d_{i}$ and $d_{j}$ is $(x, y)=\left(0,-1 / \kappa_{1}\right)$. So, intersection point of the lines $d_{i}$ and $d_{j}$ is the point $C_{1}=P-\left(1 / \kappa_{1}(P)\right) N_{P}$ in plane $S p\left\{\phi_{u}(P), N_{P}\right\}$.

Corollary 3. Directions of normals of all surfaces at a constant distance from the edge of regression on $M$ that formed along directions of $Z_{P}$ lying in plane $S p\left\{\phi_{u}, N\right\}$ intersect at a single point. This point $C_{1}=P-\left(1 / \kappa_{1}(P)\right) N_{P}$ which is referred in Theorem 2 is on the focal surface $F_{1}$.

We know that

$$
F_{1}(P)=P-\frac{1}{\kappa_{1}} N_{P}
$$

from definition of focal surfaces. Moreover, we can see easily the following equations from Figure 1:

$$
F_{1}(P)=f_{i}(P)-\mu_{i} N_{f_{i}(P)}^{f_{i}}
$$

or

$$
F_{1}(P)=f_{j}(P)-\mu_{j} N_{f_{j}(P)}^{f_{j}} .
$$

These equations show us that the focal surface $F_{1}$ of the surface $M$ can be stated by surfaces at a constant distance from 
the edge of regression on $M$ that formed along directions of $Z_{P}$ lying in plane $S p\left\{\phi_{u}, N\right\}$. If $\mu_{i}=1 / \kappa_{1}^{f_{i}}$ or $\mu_{j}=1 / \kappa_{1}^{f_{j}}$, then the focal surfaces $F_{1}$ of surfaces $M, M^{f_{i}}$, and $M^{f_{j}}$ will be the same. This case has been expressed in following theorem.

Theorem 4. Focal surfaces $F_{1}$ of the surface $M$ and surfaces at a constant distance from the edge of regression on $M$ that formed along directions of $Z_{P}$ lying in plane $S p\left\{\phi_{u}, N\right\}$ are the same if and only if first principal curvature $\kappa_{1}$ of the surface $M$ is constant.

Proof. Suppose that focal surfaces $F_{1}$ of surfaces $M$ and $M^{f}$ formed along directions of $Z_{P}$ lying in plane $\operatorname{Sp}\left\{\phi_{u}, N\right\}$ intersect; then, $\mu_{i}$ mentioned in (21) must be

$$
\mu_{i}=\frac{1}{\kappa_{1}^{f_{i}}}
$$

First principal curvature $\kappa_{1}^{f}$ of $M^{f}$ formed along directions of $Z_{P}$ lying in plane $S p\left\{\phi_{u}, N\right\}$, that is, for $\lambda_{2}=0$, is calculated by Tarakci as [1]

$$
\kappa_{1}^{f}=\frac{1}{\sqrt{\lambda_{1}^{2} \kappa_{1}^{2}+\left(1+\lambda_{3} \kappa_{1}\right)^{2}}}\left(\frac{\lambda_{1}\left(\partial \kappa_{1} / \partial u\right)}{\lambda_{1}^{2} \kappa_{1}^{2}+\left(1+\lambda_{3} \kappa_{1}\right)^{2}}+\kappa_{1}\right) .
$$

Besides, from Figure 1, since $\mu_{i}=\left|\overrightarrow{C_{1} f_{i}(P)}\right|$ is distance between points of $C_{1}=\left(0,-1 / \kappa_{1}\right)$ and $f_{i}(P)=\left(\lambda_{1}, \lambda_{3}\right)$ lying in plane $S p\left\{\phi_{u}, N\right\}$, we can write

$$
\mu_{i}=\left|\overrightarrow{C_{1} f_{i}(P)}\right|=\sqrt{\lambda_{1}^{2}+\left(\lambda_{3}+\frac{1}{\kappa_{1}}\right)^{2}}
$$

If we substitute (24) and (25) in (23) and make necessary arrangements, we obtain

$$
\frac{\partial \kappa_{1}}{\partial u}=0
$$

Thus, we have $\kappa_{1}=$ const. The converse statement is trivial. Hence, our theorem is proved.

Theorem 5. Let surface $M$ be given by parametrical $\phi(u, v)$. We consider all surfaces at a constant distance from the edge of regression on $M$ that formed along directions of $Z_{P}$ lying in plane $S p\left\{\phi_{v}, N\right\}$. Normals of these surfaces at points $f(P)$ corresponding to point $P \in M$ generate a spatial family of line of which top is center of second principal curvature $C_{2}=P-$ $\left(1 / \kappa_{2}(P)\right) N_{P}$ at $P$.

Proof. Surfaces at a constant distance from the edge of regression on $M$ that formed along directions of $Z_{P}$ lying in plane $S p\left\{\phi_{v}, N\right\}$ are defined by

$$
\begin{aligned}
& f_{i}: M \longrightarrow M^{f_{i}}, \quad i=1,2, \ldots \\
& f_{i}(P)=P+\lambda_{2_{i}} \phi_{v}(P)+\lambda_{3_{i}} N_{P} .
\end{aligned}
$$

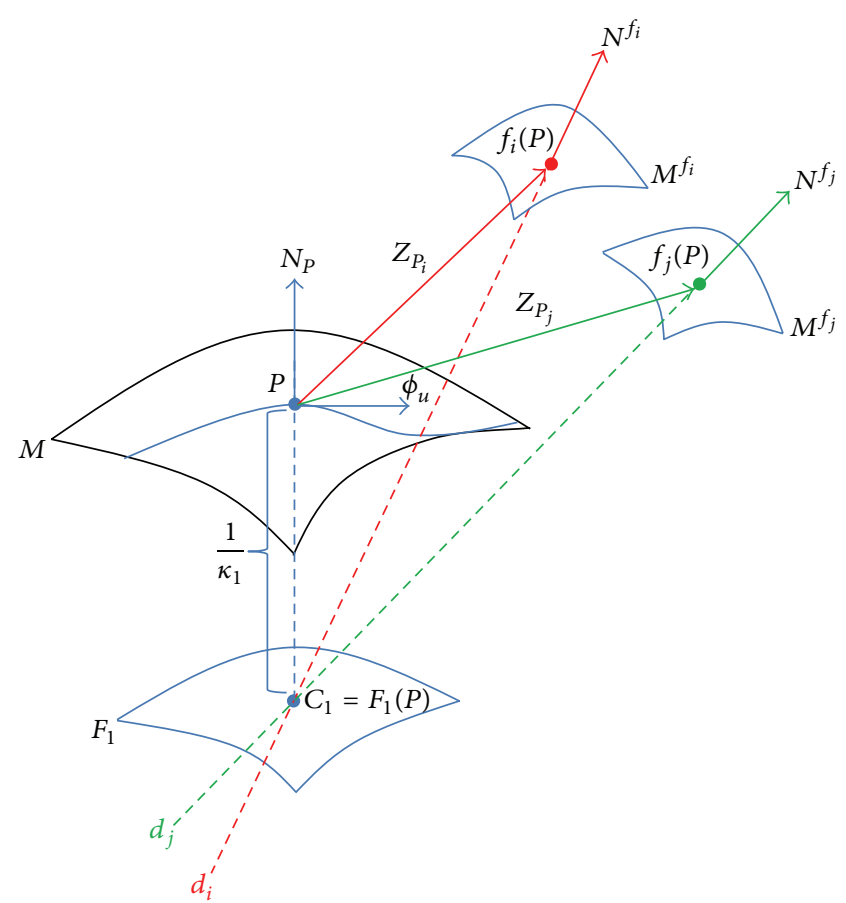

FIGURE 1: Directions of normals of all surfaces at a constant distance from the edge of regression on $M$ that formed along directions of $Z_{P}$ lying in plane $S p\left\{\phi_{u}, N\right\}$ and their intersection point (focal point).

These surfaces and their unit normal vectors are, respectively, denoted by $M^{f_{i}}$ and $N^{f_{i}}$. We will demonstrate that intersection point of lines which pass from the point $f_{i}(P)$ and are in direction $N_{f_{i}(P)}^{f_{i}}$ is $C_{2}=P-\left(1 / \kappa_{2}(P)\right) N_{P}$.

The normal vector of the surface $M^{f_{i}}$ at the point $f_{i}(P)$ is

$$
N^{f_{i}}=\lambda_{2_{i}} \kappa_{2}(P) \phi_{v}(P)+\left(1+\lambda_{3_{i}} \kappa_{2}(P)\right) N_{P}
$$

Here, it is clear that $N^{f_{i}}$ is in plane $S p\left\{\phi_{v}, N\right\}$. Suppose that line passing from the point $f_{i}(P)$ and being in direction $N_{f_{i}(P)}^{f_{i}}$ is $d_{i}$ and a representative point of $d_{i}$ is $Q=(x, y)=x \phi_{v}(P)+$ $y N_{P}$; then, equation of $d_{i}$ is

$$
d_{i} \cdots \overrightarrow{P Q}=\overrightarrow{P f_{i}(P)}+\mu_{1} N_{f_{i}(P)}^{f_{i}}
$$

Besides, suppose that line passing from the point $f_{j}(P)$ of the surface $M^{f_{j}}$ and being in direction $N_{f_{j}(P)}^{f_{j}}$ is $d_{j}$ and a representative point of $d_{j}$ is $R=(x, y)$; then, equation of $d_{j}$ is

$$
d_{j} \cdots \overrightarrow{P R}=\overrightarrow{P f_{j}(P)}+\mu_{2} N_{f_{j}(P)}^{f_{j}}, \quad j=1,2, \ldots
$$

We find intersection point of these two lines. Since it is studied in plane of vectors $\left\{\phi_{v}(P), N_{P}\right\}$, the point $P$ can be taken 
as beginning point. If we arrange the lines $d_{i}$ and $d_{j}$, then we find

$$
\begin{aligned}
d_{i} \cdots(x, y) & =\left(\lambda_{2_{i}}, \lambda_{3_{i}}\right)+\mu_{1}\left(\lambda_{2_{i}} \kappa_{1}, 1+\lambda_{3_{i}} \kappa_{2}\right), \\
d_{i} \cdots y & =\frac{1+\lambda_{3_{i}} \kappa_{2}}{\lambda_{2_{i}} \kappa_{2}} x-\frac{1}{\kappa_{2}}, \\
d_{j} \cdots(x, y) & =\left(\lambda_{2_{j}}, \lambda_{3_{j}}\right)+\mu_{2}\left(\lambda_{2_{j}} \kappa_{2}, 1+\lambda_{3_{j}} \kappa_{2}\right), \\
d_{j} \cdots y & =\frac{1+\lambda_{3_{j}} \kappa_{2}}{\lambda_{2 j} \kappa_{2}} x-\frac{1}{\kappa_{2}} .
\end{aligned}
$$

From here, it is clear that intersection point of $d_{i}$ and $d_{j}$ is $(x, y)=\left(0,-1 / \kappa_{2}\right)$. So, intersection point of the lines $d_{i}$ and $d_{j}$ is the point $C_{2}=P-\left(1 / \kappa_{2}(P)\right) N_{P}$ in plane $S p\left\{\phi_{v}(P), N_{P}\right\}$.

Corollary 6. The point $C_{2}=P-\left(1 / \kappa_{2}(P)\right) N_{P}$ which is referred in Theorem 5 is on the focal surface $F_{2}$.

Similar to Figure 1, we can write equations

$$
F_{2}(P)=f_{i}(P)-\mu_{i} N_{f_{i}(P)}^{f_{i}}
$$

or

$$
F_{2}(P)=f_{j}(P)-\mu_{j} N_{f_{j}(P)}^{f_{j}}
$$

These equations show us that the focal surface $F_{2}$ of the surface $M$ can be stated by surfaces at a constant distance from the edge of regression on $M$ that formed along directions of $Z_{P}$ lying in plane $S p\left\{\phi_{v}, N\right\}$. If $\mu_{i}=1 / \kappa_{2}^{f_{i}}$ or $\mu_{j}=1 / \kappa_{2}^{f_{j}}$, then the focal surfaces $F_{2}$ of surfaces $M, M^{f_{i}}$, and $M^{f_{j}}$ will be the same. This case has been expressed in following theorem.

Theorem 7. Focal surfaces $F_{2}$ of the surface $M$ and surfaces at a constant distance from the edge of regression on $M$ that formed along directions of $Z_{P}$ lying in plane $S p\left\{\phi_{v}, N\right\}$ are the same if and only if second principal curvature $\kappa_{2}$ of the surface $M$ is constant.

Proof. Suppose that focal surfaces $F_{2}$ of surfaces $M$ and $M^{f}$ formed along directions of $Z_{P}$ lying in plane $\operatorname{Sp}\left\{\phi_{v}, N\right\}$ intersect; then, $\mu_{i}$ mentioned in (32) must be

$$
\mu_{i}=\frac{1}{\kappa_{2}^{f_{i}}}
$$

Second principal curvature $\kappa_{2}^{f}$ of $M^{f}$ formed along directions of $Z_{P}$ lying in plane $S p\left\{\phi_{v}, N\right\}$, that is, for $\lambda_{1}=0$, is calculated by Tarakci as [1]

$$
\kappa_{2}^{f}=\frac{1}{\sqrt{\lambda_{2}^{2} \kappa_{2}^{2}+\left(1+\lambda_{3} \kappa_{2}\right)^{2}}}\left(\frac{\lambda_{2}\left(\partial \kappa_{2} / \partial \nu\right)}{\lambda_{2}^{2} \kappa_{2}^{2}+\left(1+\lambda_{3} \kappa_{2}\right)^{2}}+\kappa_{2}\right) .
$$

Besides, similar to Figure 1, since $\mu_{i}=\left|\overrightarrow{C_{2} f_{i}(P)}\right|$ is the distance between points of $C_{2}=\left(0,-1 / \kappa_{2}\right)$ and $f_{i}(P)=\left(\lambda_{2}, \lambda_{3}\right)$ lying in plane $S p\left\{\phi_{v}, N\right\}$, we can write

$$
\mu_{i}=\left|\overrightarrow{C_{2} f_{i}(P)}\right|=\sqrt{\lambda_{2}^{2}+\left(\lambda_{3}+\frac{1}{\kappa_{2}}\right)^{2}} .
$$

If we substitute (35) and (36) in (34) and make necessary arrangements, we obtain

$$
\frac{\partial \kappa_{2}}{\partial v}=0
$$

Thus, we have $\kappa_{2}=$ const. The converse statement is trivial. Hence, our theorem is proved.

Points on the surface $M$ can have the same curvature in all directions. These points correspond to the umbilics, around which local surface is sphere-like. Since normal rays of umbilic points pass through a single point, the focal mesh formed by vertices around an umbilic point can shrink into a point [11].

\section{Conflict of Interests}

The authors declare that there is no conflict of interests regarding the publication of this paper.

\section{References}

[1] Ö. Tarakci, Surfaces at a constant distance from the edge of regression on a surface [Ph.D. thesis], Ankara University Institute of Science, Ankara, Turkey, 2002.

[2] Ö. Tarakci and H. H. Hacisalihoğlu, "Surfaces at a constant distance from the edge of regression on a surface," Applied Mathematics and Computation, vol. 155, no. 1, pp. 81-93, 2004.

[3] N. Aktan, A. Görgülü, E. Özüsaglam, and C. Ekici, "Conjugate tangent vectors and asymptotic directions for surfaces at a constant distance from edge of regression on a surface," International Journal of Pure and Applied Mathematics, vol. 33, no. 1, pp. 127-133, 2006.

[4] D. Sağlam and Ö. Kalkan, "Surfaces at a constant distance from the edge of regression on a surface in $E_{1}^{3}$," Differential Geometry-Dynamical Systems, vol. 12, pp. 187-200, 2010.

[5] H. Hagen, H. Pottmann, and A. Divivier, "Visualization functions on a surface," Journal of Visualization and Animation, vol. 2, pp. 52-58, 1991.

[6] H. Hagen and S. Hahmann, "Generalized focal surfaces: a new method for surface interrogation," in Proceedings of the IEEE Conference on Visualization (Visualization '92), pp. 70-76, Boston, Mass, USA, October 1992.

[7] H. Hagen and S. Hahmann, "Visualization of curvature behaviour of free-form curves and surfaces," Computer-Aided Design, vol. 27, no. 7, pp. 545-552, 1995.

[8] H. Hagen, S. Hahmann, T. Schreiber, Y. Nakajima, B. Wordenweber, and P. Hollemann-Grundstedt, "Surface interrogation algorithms," IEEE Computer Graphics and Applications, vol. 12, no. 5 , pp. 53-60, 1992. 
[9] J. Hoschek, Linien-Geometrie, BI, Wissensehaffs, Zurich, Switzerland, 1971.

[10] K. Strubecker, Differentialgeometrie III, De Gruyter, Berlin, Germany, 1959.

[11] J. Yu, X. Yin, X. Gu, L. McMillan, and S. Gortler, "Focal Surfaces of discrete geometry," in Eurographics Symposium on Geometry Processing, 2007. 


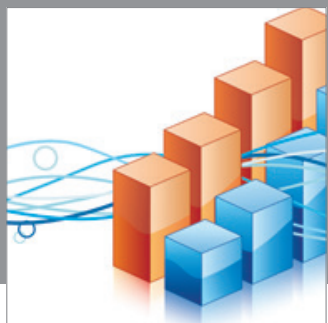

Advances in

Operations Research

mansans

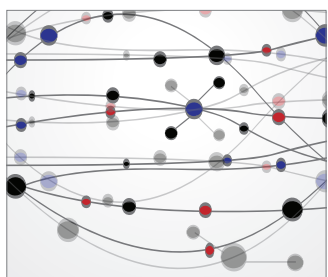

The Scientific World Journal
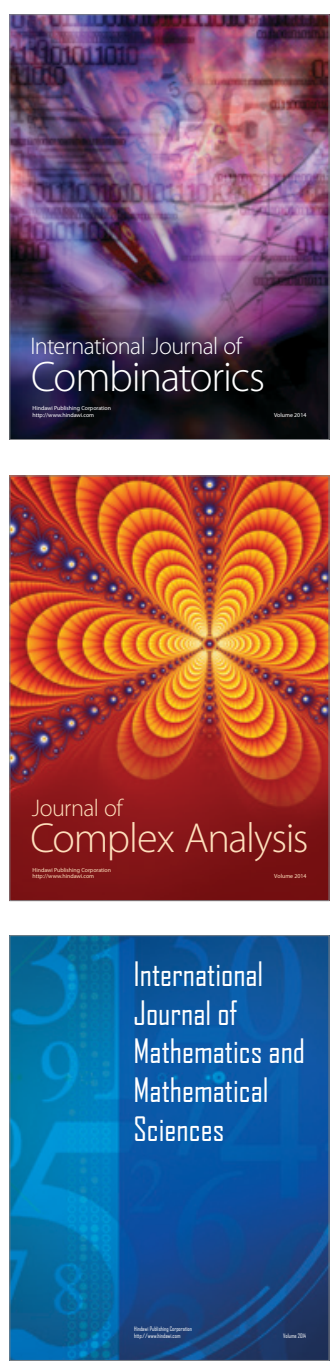
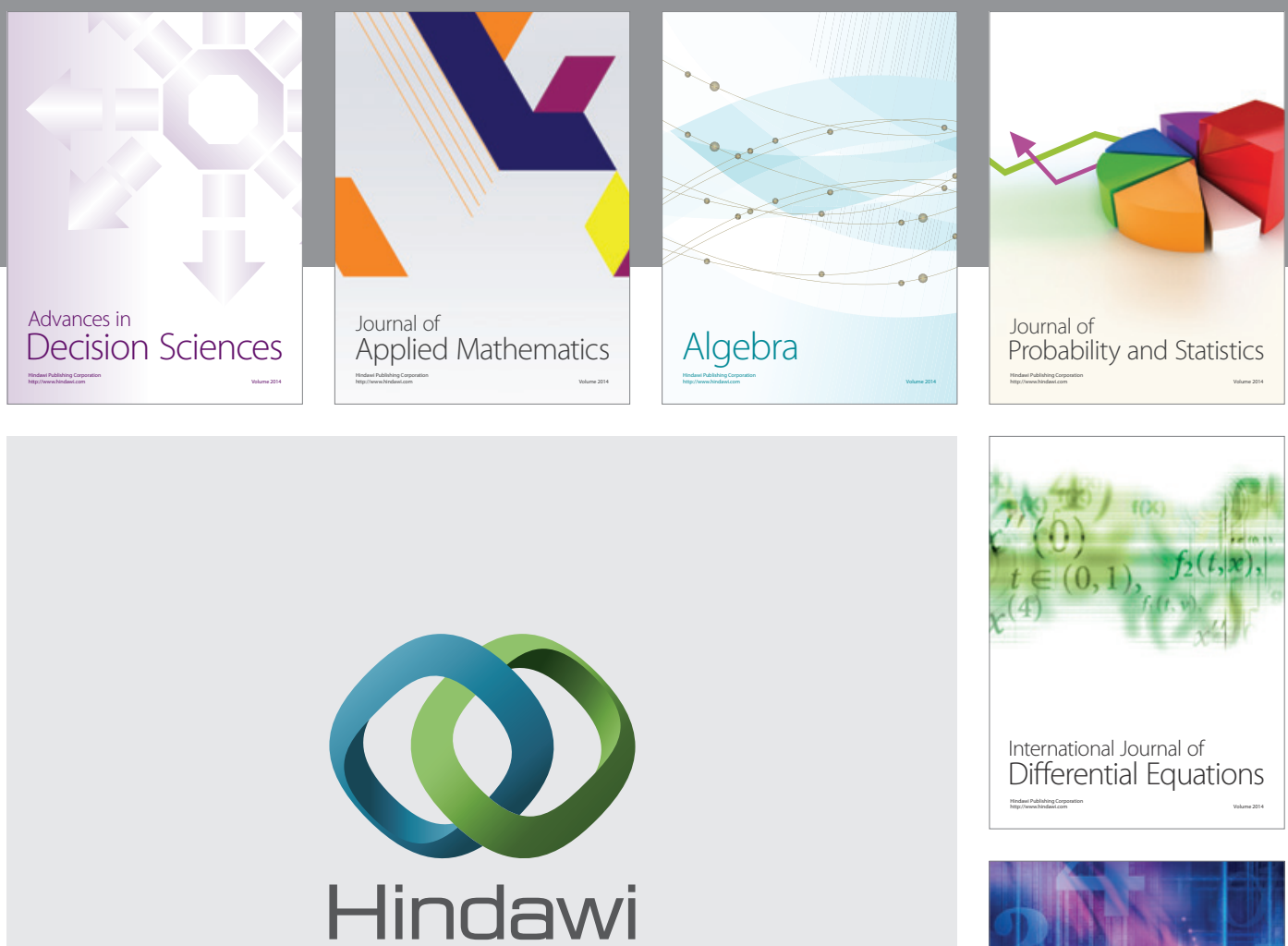

Submit your manuscripts at http://www.hindawi.com
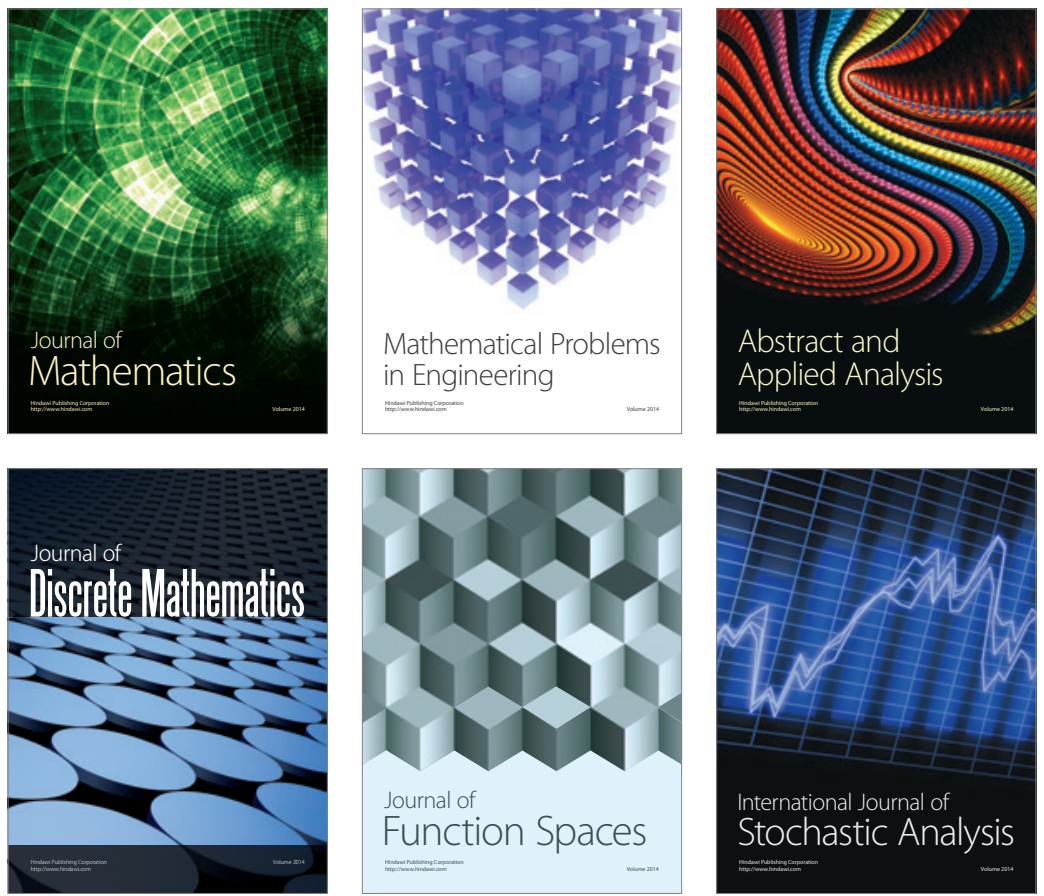

Journal of

Function Spaces

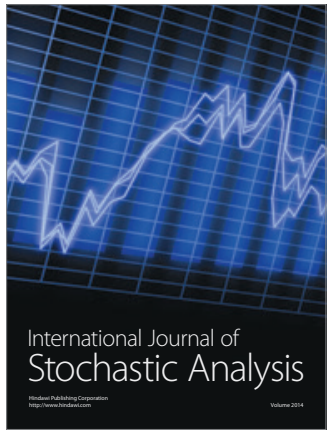

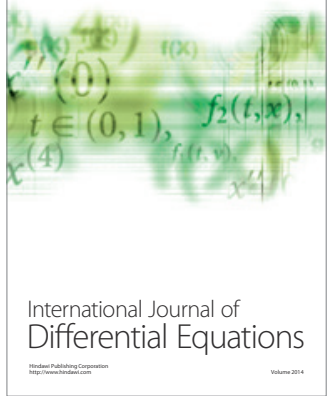
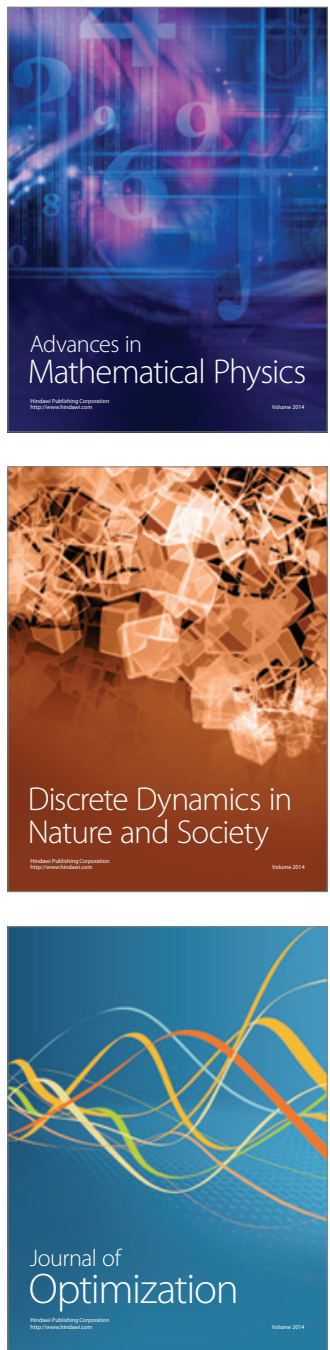